\title{
Characterizing Functional Integrity: Intraindividual Brain Signal Variability Predicts Memory Performance in Patients with Medial Temporal Lobe Epilepsy
}

\author{
Andrea B. Protzner, ${ }^{1,2}$ Natasa Kovacevic, ${ }^{3}$ Melanie Cohn, $, 4,5$ and Mary Pat McAndrews ${ }^{4,5}$ \\ ${ }^{1}$ Department of Psychology, University of Calgary, Canada, ${ }^{2}$ Hotchkiss Brain Institute, Canada, ${ }^{3}$ Rotman Research Institute, Canada, ${ }^{4}$ Krembil \\ Neuroscience Centre, University Health Network, Canada, and ${ }^{5}$ Department of Psychology, University of Toronto, Canada
}

Computational modeling suggests that variability in brain signals provides important information regarding the system's capacity to adopt different network configurations that may promote optimal responding to stimuli. Although there is limited empirical work on this construct, a recent study indicates that age-related decreases in variability across the adult lifespan correlate with less efficient and less accurate performance. Here, we extend this construct to the assessment of cerebral integrity by comparing fMRI BOLD variability and fMRI BOLD amplitude in their ability to account for differences in functional capacity in patients with focal unilateral medial temporal dysfunction. We were specifically interested in whether either of these BOLD measures could identify a link between the affected medial temporal region and memory performance (as measured by a clinical test of verbal memory retention). Using partial least-squares analyses, we found that variability in a set of regions including the left hippocampus predicted verbal retention and, furthermore, this relationship was similar across a range of cognitive tasks measured during scanning (i.e., the same pattern was seen in fixation, autobiographical recall, and word generation). In contrast, signal amplitude in the hippocampus did not predict memory performance, even for a task that reliably activates the medial temporal lobes (i.e., autobiographical recall). These findings provide a powerful validation of the concept that variability in brain signals reflects functional integrity. Furthermore, this measure can be characterized as a robust biomarker in this clinical setting because it reveals the same pattern regardless of cognitive challenge or task engagement during scanning.

\section{Introduction}

Considerable effort has been put toward identifying an fMRI measure that can serve as a reliable biomarker of functional integrity in disorders that affect the medial temporal lobe (MTL), such as Alzheimer's Disease (AD), and medial temporal-lobe epilepsy (mTLE). Although much of the work focuses specifically on MTL activation, more recent studies emphasize functional connectivity in networks including the default mode network, where changes in intrinsic activity dynamics correlate with clinical indices of pathology, such as seizure duration and cognitive dysfunction (Zhang et al., 2010; Pihlajamäki and Sperling, 2009; Zhang and Raichle, 2010).

Regardless of the imaging tasks used, most brain imaging studies examining disease pathophysiology focus on signal amplitude to characterize brain changes. However, recent computational modeling suggests that variability in physiological signals also is an important parameter reflecting the processing capacity

\footnotetext{
Received June 25, 2012; revised May 1, 2013; accepted May 3, 2013.

Author contributions: A.B.P. and M.P.M. designed research; M.C. and M.P.M. performed research; N.K. contributed unpublished reagents/analytic tools; A.B.P. and N.K. analyzed data; A.B.P., M.C., and M.P.M. wrote the paper.

This work was supported by grants from the James S. McDonnell Foundation (JSMF No. 22002055) and the Canadian Institutes of Health Research (MOP 97891) to M.P.M. We thank Filomeno Cortese for his help with data illustration.

Correspondence should be addressed to Dr Andrea B. Protzner, Department of Psychology, University of Calgary,2500 University Drive NW, Calgary, Alberta, Canada, T2N 1N4. E-mail: protzner@ucalgary.ca.

DOI:10.1523/JNEUROSCI.3009-12.2013

Copyright $\odot 2013$ the authors $\quad 0270-6474 / 13 / 339855-11 \$ 15.00 / 0$
}

and functional integrity of biological systems (Deco et al., 2011). In a complex nonlinear system such as the brain, variability facilitates the transition from one network configuration to another in dealing with ambiguous environmental signals. It enables the system to explore multiple states and thus generate multiple behaviors. In essence, variability provides the kinetic energy that allows the brain to respond flexibly to the environment (McIntosh et al., 2010).

There are some empirical studies to support this computational view. In an aging study, Garrett et al. (2011) examined BOLD signal variability measured during simple cognitive tasks. They found that younger, faster, more consistent performers exhibited significantly higher brain variability compared with older, poorer-performing adults. Montez et al. (2009), using MEG, reported that changes in variability, as characterized by changes in temporal autocorrelations, were correlated with cognitive deficits in patients with mild AD. With mTLE patients undergoing intracranial electroencephalography (iEEG), our group and others found reduced variability in epileptogenic tissue as demonstrated by decreased multiscale entropy or increased temporal autocorrelations (Parish et al., 2004; Monto et al., 2007; Protzner et al., 2010). Thus, neurocognitive and neurophysiological operations may depend on an optimal amount of internal variability and this can be disrupted by both focal and more global brain changes (Frank et al., 1999).

Here, we compare these two different characteristics in BOLD signal, mean amplitude, and variability, to see which best predicts 
functional integrity (operationalized as "percent retained" on a standard clinical verbal memory test) in patients with mTLE. We used percent retained as our criterion indicator because it is a well established clinical marker of left mesial temporal integrity in this population (Trenerry et al., 1993; Sawrie et al., 2001). Brain signal was captured by BOLD activity on an experimental paradigm involving autobiographical memory (AM), which has been shown to engage the hippocampus (Svoboda et al., 2006; Addis et al., 2007; McDermott et al., 2009) and language imaging protocols used for presurgical planning. Using multiple tasks during scanning allowed us to examine the generalizability of our putative indices of functional integrity; (1) by focusing on an AM task that is selective to hippocampal engagement, and (2) by examining differences between task-positive (language) and tasknegative (fixation) states.

\section{Materials and Methods \\ Participants}

Twenty-three individuals with pharmacologically intractable mTLE who were evaluated for surgical candidacy at the Epilepsy program at Toronto Western Hospital, University Health Network were included in this study. Ten patients had left MTL seizures (6 male, 4 female; mean age, 30.3 years; range $=18-47$ years), and 13 had right MTL seizures ( 4 male, 9 female; mean age, 38.2 years; range $=18-62$ years) based on scalp or (in one case) intracranial EEG recordings from their admission to the Epilepsy Monitoring Unit. Structural MRI revealed medial temporal lobe sclerosis (MTS) on the side of the epileptic focus in six left mTLE patients and six right mTLE patients. All patients had language lateralized to the left hemisphere based on lateralization indices derived from a panel of fMRI activation tasks (verb generation, category fluency). Finally, all were on a combination of two to four antiepileptic drugs at the time of fMRI and neuropsychological testing, but none had good seizure control which was the reason for consideration of surgery. Exclusion criteria included epileptogenic foci or lesions outside of the MTL (e.g., frontal or temporal neocortical) and structural lesions other than MTS. This research project was approved by University Health Network Research Ethics Board.

\section{fMRI tasks}

We used an experimental memory paradigm (conditions are autobiographical memory and sentence completion) that was run concurrently with the scans included in our standard language lateralization protocol for presurgical assessment (conditions are category fluency, verb generation, and fixation). Autobiographical memory and sentence completion were scanned in an event-related paradigm, and the remaining tasks were scanned in blocked design paradigms. No overt responses were collected during scanning; participants were instructed to generate words or retrieve memories silently as long as the cue remained visible. Stimuli for all tasks were presented as black text on a white background. Detailed instructions and practice items were given before scanning to ensure each participant's comprehension.

During the autobiographical memory task, participants saw generic event titles (e.g., first kiss) as prompts to retrieve a relevant autobiographical memory. Each of 20 autobiographical memory trials lasted $12 \mathrm{~s}$, comprised of an event title presented for $6 \mathrm{~s}$, followed by $6 \mathrm{~s}$ of passive viewing of symbols (e.g., +++++++ or ${ }^{\star * \star * \star *}$ ). A brief postscan interview was completed at the end of the scanning session to verify retrieval success. All participants were able to retrieve specific memories for most cues (i.e., a minimum 18/20).

The autobiographical memory control task was sentence completion, where participants completed a sentence with a word, thereby retrieving semantic information. This task controlled for the narrative processes and semantic retrieval inherent in AM retrieval, which are relatively spared in MTL damage (Addis et al., 2007; St-Laurent et al., 2009). Participants saw a sentence with the last word missing (e.g., "The dog ate a

.") and were instructed to complete the sentence silently. Each of 20 sentences were presented for $4 \mathrm{~s}$, followed by $8 \mathrm{~s}$ of passive viewing of symbols (e.g., +++++++ or ${ }^{* * * * * * *}$ ). AM and sentence completion trials were intermixed, and presented in random order.

Category fluency incorporated common noun generation, proper name generation and fixation blocks. For the common noun generation, participants saw category names (e.g., fruits) to which they covertly generated common noun exemplars (e.g., apples, bananas, etc). For the proper nouns block, participants saw a category name with stars on both sides (e.g., ${ }^{*}$ countries $^{*}$ ) to which they covertly generated proper noun exemplars (e.g., Canada, USA, etc.). Fixation blocks required passive viewing of symbols (e.g., +++++++ or ${ }^{* * * * * * *}$ ). The duration of each common noun generation, proper name generation and fixation block was $25 \mathrm{~s}$, and was comprised of two stimuli, presented for $12 \mathrm{~s}$, with a $500 \mathrm{~ms}$ interstimulus interval; there were six repetitions of the paradigm during the scan.

For verb generation, participants saw either nouns (e.g., glass) to which they covertly generated related verbs (e.g., break, clean, sip), or fixation symbols (e.g., ${ }^{\star} \#^{\star} \#^{*}$ ) which required passive viewing. Both verb generation and fixation blocks lasted $27 \mathrm{~s}$, and were comprised of five stimuli, presented for $5 \mathrm{~s}$, with a $500 \mathrm{~ms}$ interstimulus interval; there were 10 repetitions of the paradigm during the scan.

\section{Behavioral measures}

Two behavioral measures from the standard preoperative clinical neuropsychological assessment were included. The first is an indicator of verbal memory retention from the Rey Auditory Verbal Learning Test (RAVLT) (Rey, 1964). The RAVLT is a word-list learning task in which participants recall a 15-word list over five repeated study test trials and again following the presentation of an interfering list and following a 20 min delay filled with other tasks. Percent retained is the number of words recalled after the 20 min delay divided by the number of words recalled immediately after the fifth learning trial.

In the context of mTLE, we were concerned with predicting significant risk to memory function (particularly verbal memory, which is a more salient domain for patients) should this region be surgically removed in an attempt to cure seizures. Although a number of neuropsychological measures are used in our formulation regarding risk, the principal one we rely on in our practice is percent retained. Thus, we hypothesized that hippocampal functional integrity would correlate with percent retained scores, making this measure our "gold standard" for biomarker utility.

We additionally used a second behavioral measure, the Full Scale Intelligence Quotient (FSIQ) from the Wechsler Abbreviated Scale of Intelligence (Wechsler, 1999). FSIQ (as estimated by the WASI) is a variable that expresses complex coordinated functioning of many brain networks. It is a reflection of both "crystallized" components based on semantic information as well as "fluid" components that reflect online problemsolving. Hence it is much less dependent on the integrity of a focal region, such as the MTL, and not found to be at risk for decline with temporallobe surgery for epilepsy (Sherman et al., 2011). We therefore hypothesized that that FSIQ would not vary systematically across our group of patients in relation to their epilepsy or their at-risk function (verbal memory) and could thus serve as a control variable to assess the specificity of our findings.

We used behavioral criterion measures that were collected outside the context of scanning for two reasons. First, the crucial question we were trying to address is whether amplitude and variability are "general" biomarkers for MTL integrity and in this context we considered it important to link our findings to standard clinical measures used in surgical decision-making in the mTLE population. The second reason was also related to our pursuit of generalizability. Specifically, we wanted to test the robustness of these relationships between brain signal and clinical memory variables using standard clinical imaging paradigms (such as our language lateralization protocols) as well as one used in our ongoing research on autobiographical memory. None of these paradigms employed behavioral measures.

\section{Data acquisition}

Anatomical and functional data were acquired on a 3-T Signa MR System (GE Medical Systems). Functional data were acquired in an interleaved order (TR $=2 \mathrm{~s} ; 28$ slices or 32 slices determined by head size, $240 \mathrm{~mm}$ 
FOV, $64 \times 64$ matrix, resulting in a voxel size of $3.75 \times 3.75 \times 5.0)$. Scans were taken in an oblique orientation that was orthogonal to the long axis of the hippocampus to maximize signal intensity and minimize partial volume effects in the MTL. Three-dimensional anatomical scans were acquired with higher spatial resolution (T1-weighted sequence, 120 slices, $220 \mathrm{~mm}$ FOV, $256 \times 256$ matrix, resulting in a voxel size of $0.78,125 \times 0.78,125 \times 1.0 \mathrm{~mm})$.

\section{Data preprocessing}

In two recent papers by Garrett et al. $(2010,2011)$ brain signal variability was examined in relation to aging. We used the same preprocessing pipeline, the details of which are given below.

We created an unbiased nonlinear group average anatomical image (Kovacevic et al., 2005; Chen et al., 2006; Levine et al., 2008; Garrett et al., 2010), which we refer to as the Common Template. The anatomical scan for each patient was nonlinearly registered to the Common Template. Functional data were slice-time corrected using AFNI (http://afni.nimh. nih.gov/afni) and motion corrected using AIR (http://bishopw.loni.ucla. edu/AIR5/) by registering all functional volumes to the 100th volume within each run. By averaging all functional volumes within a motioncorrected run, we calculated mean functional volumes. For each run, the mean functional volume was registered with each subject's structural image using a rigid body transformation model. After appropriate transform concatenations, from initial volume to the 100th volume within run, from mean run volume to structural volume, and from structural volume into the Common Template space, we obtained a direct nonlinear transform from each initial fMRI volume into the Common Template space. We then applied the FSL/FNIRT registration algorithm to find a nonlinear transform between our anatomical Common Template and MNI 152_T1 provided with FSL software (www.fmrib.ox.ac.uk/fsl).

Due to our focus on variability in brain signal, we performed several additional preprocessing steps to reduce data artifacts. Functional volumes in the Common Template space were corrected for artifacts via independent component analysis (ICA) within separate runs, as implemented in FSL/MELODIC (Beckmann and Smith, 2004). The ICA denoising procedure was performed with an in-house classifier, very similar to that used by Tohka et al. (2008). We randomly selected seven right and left mTLE patients. One of the authors (A.B.P.) manually identified artifactual components for one run per subject. Each component was visually evaluated based on combined information from spatial distribution, time series, and spectral power distribution. We then defined a set of features that captured the most common characteristics of artifacts, such as focality of the spatial distribution, ratio of weights within ventricles versus brain, ratio of weights along the outer edge of the brain versus the rest of the brain, relative spectral power distribution within low, medium and high-frequency ranges, and presence of spikes within the time course. A quadratic classifier based on the evaluated features was trained using manually classified ICA decompositions. The classifier was applied to the remainder of the data for all subjects and runs, and all components were classified as either artifact or nonartifact. By subtracting the signal from the artifactual components (using fsl_regfilt function from FSL) we extracted denoised fMRI time series. Because we used a conservative approach during identification of artifacts, voxel time series were further adjusted by regressing out motion correction parameters, white matter (WM), and CSF time series. For WM and CSF regression, we extracted time series from unsmoothed data within small ROIs in the corpus callosum and ventricles of the Common Template, respectively. ROIs were selected such that they were deep within each structure of interest (corpus callosum and ventricles) to avoid signal contamination from external tissues due to misregistration and partial volume effects. The rationale for using small ROIs and unsmoothed data was to ensure that the ROIs would not contain any signal of interest (i.e., gray matter signal) for any of the subjects. The choice of a single $4 \mathrm{~mm}^{3}$ voxel within corpus callosum for WM and a same-size voxel within one lateral ventricle for CSF was based on our experience in having excellent registration of these structures. Finally, voxel signal was spatially smoothed using $8 \mathrm{~mm}$ Gaussian kernel.

We localized gyral locations and Brodmann areas from our functional output by reference to Talairach and Tournoux (1988) after transform- ing MNI coordinates to Talairach space with the Nonlinear Yale MNI to Talairach Conversion Algorithm (Lacadie et al., 2008) and associated online Java-based applet.

\section{Data analyses}

Calculation of BOLD signal mean and SD. To calculate BOLD signal mean for each task, we first expressed signal value as a percentage change from its respective block onset value. Tasks collected during the event-related paradigm (autobiographical memory and sentence completion) consisted of long trials, and were transformed into mini-blocks for the purpose of these analyses. We then calculated a mean percentage change within each block and averaged across all blocks. To calculate BOLD signal SD, we normalized all blocks for each task such that the overall four-dimensional mean across brain and block was 100. For each voxel, we subtracted the block mean and concatenated within-task across all blocks. Finally, we calculated within-task voxel SDs across this concatenated mean-block corrected time series.

Partial least-squares analyses. For each of the two fMRI signal measures, SD and mean, we performed separate partial least-squares (PLS) analyses (http://rotman-baycrest.on.ca/index.php?section=84) (McIntosh et al., 1996). Additionally, we performed separate analyses for tasks presented in event-related versus blocked design paradigms.

Task PLS. We performed Task PLS on mean fMRI signal only, to confirm task engagement. Task PLS uses singular value decomposition to identify distributed activity patterns, or latent variables (LVs), that show similarities or differences between experimental conditions. Each LV contains three vectors. The first vector contains a singular value, which indicates the strength of the effect expressed by the LV. The remaining two vectors relate and experimental design and brain activity. The experimental design vector contains task saliences, which indicate the degree to which each task is related to the brain signal pattern identified in the LV. These task saliences can be interpreted as the optimal contrast that codes the effect depicted in the LV. The brain signal vector contains voxel saliences. These are numerical voxel weights that identify the collection of voxels that, as a whole, are most related to the effects expressed in the LV. PLS is similar to other multivariate techniques, such as principal component analysis, in that the algorithm extracts LVs explaining the covariance between conditions and brain activity in order of the amount of covariance explained, with the LV accounting for the most covariance extracted first.

Behavior PLS. As our primary interest was patterns of brain activity that explained variation in memory performance, we used behavior PLS to examine task-dependent correlations between our behavior measures (percent retained and FSIQ) and voxel signal (either BOLD mean or BOLD SD) throughout the brain (for a detailed description of PLS's application to SD in blocked design fMRI data) (Garrett et al., 2010).

Although our behavior measure of interest was delayed verbal retention (percent retained), which typically is associated with left medial temporal lobe integrity (Hermann et al., 1994; Miller et al., 1993; Milner, 1971), we focus on PLS analyses that were performed across all patients regardless of group. Although the typical pattern of poorer performance in left mTLE than right mTLE patients was seen in our sample, there was overlap in the distribution of scores, allowing us to exploit the full range of performance across the sample in the multivariate analysis. In essence, we were interested in characterizing the relationship between brain patterns and performance and not any particular link to the side of seizure focus. However, to ensure that our results would hold in each group considered independently, we additionally performed the analyses with left and right mTLE patients separated into two groups.

For the behavioral PLS analyses, we first created a correlation matrix, comprised of the covariance between voxel signal and measures of behavior across participants. Singular value decomposition of this matrix produces a set of distributed activity patterns, or LVs that show similarities or differences between experimental conditions and brain-behavior correlations. Each LV contains three vectors. The first vector contains a singular value, which indicates the strength of the effect expressed by the LV. The remaining two vectors relate experimental design and brain signal. The experimental design vector contains task saliences, which 
A

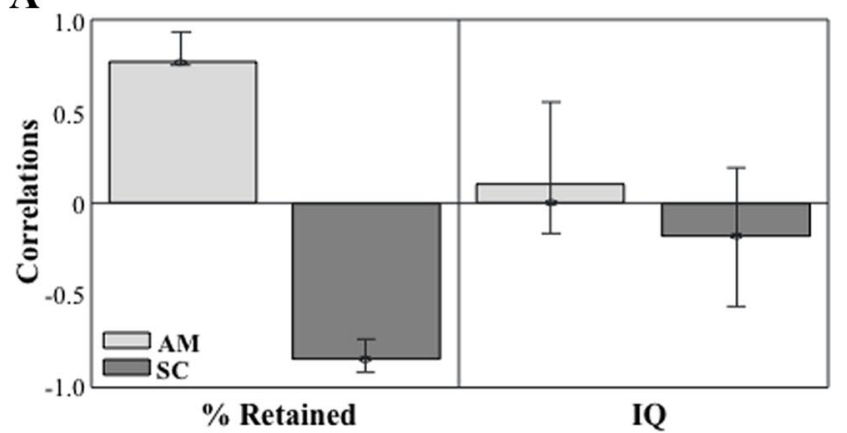

B

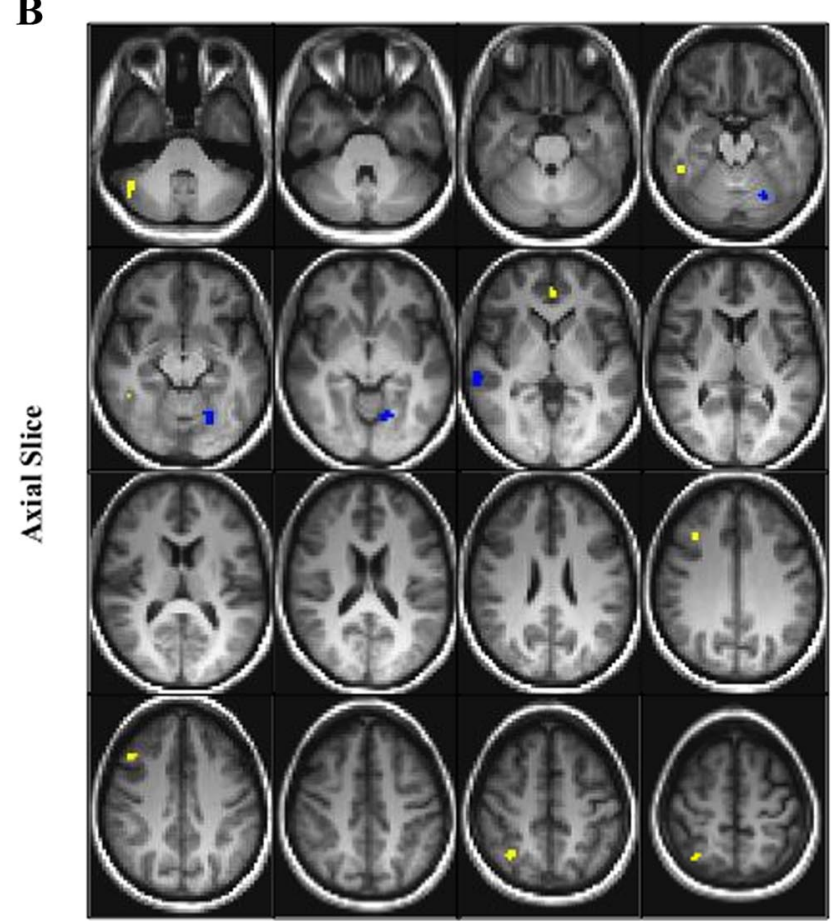

Figure 1. $\quad A$, Correlation bar graph and singular image $(B)$ for LV1 from the mean fMRI Signal in autobiographical memory and sentence completion behavior-PLS analysis. The correlation bar graph $(\boldsymbol{A})$ captures the task-dependent correlations between our behavior measures (percent retained and IQ) and the regions identified in the singular image. The error bars show the 95\% confidence interval derived from bootstrap estimation. The error bar crosses zero for IQ scores in both AM and SC tasks, indicating that there is no stable contribution from IQ to the pattern identified in the singular image. $\boldsymbol{B}$, The singular image shows brain-behavior correlations for AM and SC, displayed on axial slices in MNI atlas space. The brain is displayed according to radiological convention $(\mathrm{L}=\mathrm{R})$. Regions highlighted in in yellow indicate a positive correlation between increased brain activity during AM and better verbal memory performance. Regions highlighted in blue indicating a positive correlation between increased brain activity during SC and better verbal memory performance. SC, Sentence completion.

indicate the degree to which each task is related to the brain-behavior correlation pattern identified in the LV. The brain signal vector contains voxel saliences. These are numerical voxel weights that as a whole identify a brain pattern that optimally relates brain signal to the correlation effect expressed in the LV. Note that for each LV, there is one salience per voxel that applies for all behavior measures and tasks.

Statistical assessment. Statistical assessment for PLS was done using permutation tests for the LVs and bootstrap estimation of SEs for the voxel saliences. The permutation test assesses whether the effect represented in a given LV, captured by the singular value, is sufficiently strong to be different from random noise (McIntosh et al., 1996). This was accomplished using sampling without replacement to reassign the order of conditions for each subject. PLS was recalculated for each sample, and the number of times the permuted singular values exceeded the observed
Table 1. Local maxima for the mean fMRI signal in autobiographical memory and sentence completion behavior PLS analysis

\begin{tabular}{lrrrr}
\hline Region & \multicolumn{1}{c}{$x$} & \multicolumn{1}{c}{$y$} & \multicolumn{1}{c}{ BSR } \\
\hline Right middle temporal gyrus (BA 37) & 39 & -45 & -6 & 4.18 \\
Right middle frontal gyrus (BA 8) & 42 & 30 & 36 & 4.95 \\
Right superior frontal gyrus (BA 6) & 15 & 21 & 66 & 4.19 \\
Right superior parietal lobule (BA 7) & 30 & -54 & 51 & 4.05 \\
Left dorsomedial frontal gyrus (BA 10) & -3 & 51 & 0 & 4.54 \\
Left lingual gyrus (BA 18) & -24 & -60 & -6 & -5.38 \\
Right superior temporal gyrus (BA 42) & 57 & -24 & 3 & -4.31 \\
\hline
\end{tabular}

Only voxels in supratentorial regions are reported. Regions indicate the gyral locations and BA associated with the cluster peak.

$x, y$, and $z$ indicate voxel coordinates in MNI space. BSR represents each voxel's PLS parameter estimate divided by its SE.

singular values was calculated. Exact probabilities are presented for all LVs.

The SE estimates of the voxel weights/saliences from the bootstrap tests (i.e., bootstrap ratios) were used to assess the reliability of the nonzero voxel saliences in significant LVs. Bootstrap tests were generated using sampling with replacement, keeping the assignment of experimental conditions fixed for all subjects. PLS was recalculated for each bootstrap test. A salience whose value depends greatly on which subjects are in the sample is less precise than one that remains stable regardless of the sample chosen (Sampson et al., 1989). No corrections for multiplecomparisons are necessary because the voxel saliences are calculated in a single mathematical step on the whole brain. The bootstrap ratio is proportional to a $z$-score, but should be interpreted as a confidence interval. For this paper, we designated a threshold of 3.4 corresponding approximately to a $99.9 \%$ confidence interval, or a $p$ value $<0.001$. We used different bootstrap thresholds to capture visually all the stable voxel clusters, but keep these clusters small enough that they are easy to differentiate. Our minimum cluster size was 10 voxels. Some clusters may appear smaller in our figures because we do not display every slice, and clusters that meet our criterion via spatial contiguity in the $z$-plane may not be evident.

To ensure that our additional preprocessing steps for variability analyses successfully removed motion artifact, we calculated an average head motion index for each participant (i.e., mean framewise displacement) (Power et al., 2012). We then covaried out this motion measure from our data, as previously described (Pedhazur, 1982; McIntosh and GonzalezLima, 1991; O'Neil et al., 2012), and replicated our SD analysis on the residualized dataset. In addition, to ensure that our results were not influenced by age, we covaried out age, and again replicated our SD analysis on the residualized dataset. We found no effect of either motion or age; that is, the correlation between the bootstrap ratio thresholded results from the dataset using the preprocessing pipeline described by Garrett et al. $(2010,2011)$ and the residualized dataset ranged from 0.96 to 0.99 . Given that the spatial distribution of the effects overlap between the datasets (i.e., the effects of interest held in all cases), we elected to present the data using the preprocessing pipeline already published for SD analyses by Garrett et al., (2010, 2011).

\section{Results}

Neuropsychological data

Scores on FSIQ and RAVLT percent retained, the criteria measures in the behavioral PLS analyses, were compared between left and right mTLE. We distinguish between these groups here because there is a clear expectation that they would differ on the verbal retention measure. That was indeed the case; for RAVLT percent retained $($ left mean $=47.7, \mathrm{SD}=21.3$; right mean $=$ $\left.78.4, \mathrm{SD}=17.5 ; t_{(21)}=3.8 ; p=0.001\right)$. According to agereferenced norms, the right $\mathrm{mTLE}$ patients are relatively unimpaired and the left mTLE group shows a substantial deficit (left mean $z=-3.49, \mathrm{SD}=1.72$; right mean $z=-0.98$, $\mathrm{SD}=1.38)$. Note that percent retained is a meaningful index of memory as both groups recalled $\sim 75 \%$ of list items on the 
A

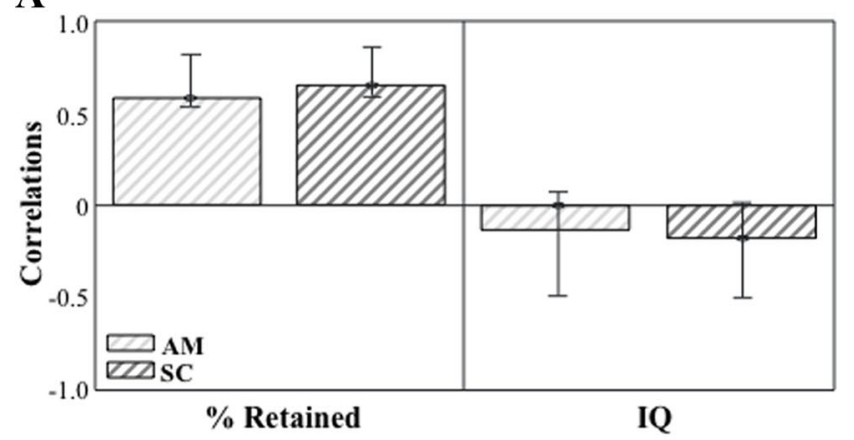

B

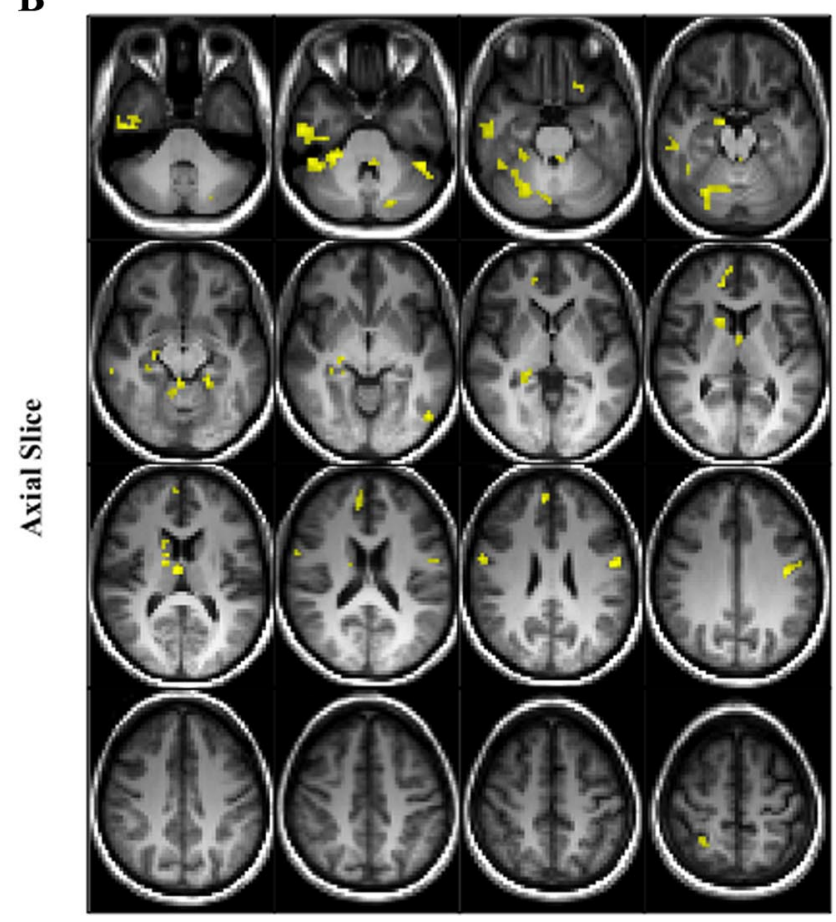

Figure 2. Correlation bar graph $(\boldsymbol{A})$ and singular image $(\boldsymbol{B})$ for LV1 from the SD in fMRI signal in autobiographical memory and sentence completion behavior-PLS analysis. The correlation bar graph $(\boldsymbol{A})$ captures the task-dependent correlations between our behavior measures (percent retained and IQ) and the regions identified in the singular image. The error bars show the $95 \%$ confidence interval derived from bootstrap estimation. The error bar crosses zero for IQ scores in both AM and SC tasks, indicating that there is no stable contribution from IQ to the pattern identified in the singular image. The singular image $(\boldsymbol{B})$ shows brain signal variabilitybehavior correlations for AM and SC, displayed on axial slices in MNI atlas space. The brain is displayed according to radiological convention $(\mathrm{L}=\mathrm{R})$. Regions highlighted in in yellow indicate a positive correlation between brain variability in both tasks and memory performance.

fifth learning trial. Thus there is no issue of a "floor effect" influencing our retention measure. There was no difference between the groups for FSIQ (left mean $=98.8, \mathrm{SD}=11.2$; right mean $\left.=101.4, \mathrm{SD}=11.9 ; t_{(21)}=0.6 ; p=0.60\right)$.

Task PLS analyses

We performed Task PLSs on mean fMRI signal to confirm task engagement. Because activations are completely in line with the published literature from ours and other labs, we do not display them in figures. In the autobiographical memory and sentence completion analysis, we identified one significant LV, differentiating brain activity during autobiographical memory from sentence completion $(p<0.001)$. Importantly, we found preferential autobiographical memory activation in typical regions, including the medial temporal
Table 2. Local maxima for the standard deviation of fMRI signal in autobiographical memory and sentence completion behavior PLS analysis

\begin{tabular}{lrrrr}
\hline Region & \multicolumn{1}{c}{$x$} & \multicolumn{1}{l}{$z$} & BSR \\
\hline Right inferior temporal gyrus (BA 21) & 48 & 6 & -36 & 6.27 \\
Right inferior temporal gyrus (BA 20) & 45 & -12 & -33 & 6.90 \\
Right temporal pole (BA 38) & 36 & 27 & -24 & 5.71 \\
Right hippocampus & 9 & -6 & -15 & 7.52 \\
Right putamen & 27 & -3 & -6 & 5.71 \\
Right thalamus & 0 & -6 & 15 & 6.56 \\
Right caudate nucleus & 12 & 0 & 18 & 6.28 \\
Right anterior cingulate (BA 32) & 9 & 48 & 9 & 5.44 \\
Right anterior cingulate (BA 24) & 3 & -3 & 27 & 6.60 \\
Right inferior frontal gyrus (BA 44) & 54 & 3 & 27 & 5.72 \\
Right medial frontal gyrus (BA 9) & 3 & 54 & 27 & 6.01 \\
Right superior parietal lobe (BA 7) & 24 & -45 & 60 & 5.33 \\
Left fusiform gyrus (BA 20) & -36 & -6 & -42 & 6.01 \\
Left inferior temporal gyrus (BA 37) & -54 & -66 & -3 & 6.76 \\
Left parahippocampal gyrus (BA 28) & -27 & -6 & -36 & 5.77 \\
Left parahippocampal gyrus (BA 30) & -24 & -39 & -9 & 6.03 \\
Left hippocampus & -30 & -30 & -3 & 5.19 \\
Left thalamus & -9 & -30 & 9 & 7.19 \\
Left inferior frontal gyrus (BA 47) & -27 & 24 & -21 & 5.41 \\
Left cuneus (BA 18) & -21 & -96 & 12 & 5.87 \\
\hline
\end{tabular}

Only voxels in supratentorial regions are reported. Regions indicate the gyral locations and BA associated with the cluster peak.

$x, y$, and $z$ indicate voxel coordinates in MNI space. BSR represents each voxel's PLS parameter estimate divided by its SE.

regions in evidence (data from a subset of these patients are published in McAndrews and Cohn, 2012). We found preferential sentence completion activation in a set of regions including left inferior prefrontal cortex, posterior middle temporal cortex, and inferior parietal lobe.

For the fixation, verb generation, and category fluency analysis, we identified one significant $L V$, differentiating brain activity during fixation versus verb generation and category fluency $(p<0.001)$. We found preferential language-related activation in a canonical left-lateralized set of regions in frontal and temporal-parietal lobes. This LV did not reveal many of the regions that have been associated with the default mode network (Fox et al., 2005) during fixation, but we did find preferential fixation-related activity primarily in a dorsal region of the posterior cingulate cortex as well as right posterior temporal lobe. Finally, as part of our standard clinical practice for presurgical language localization, we performed univariate analyses with AFNI (http://afni.nimh.nih.gov/) (Cox, 1996) on each patient individually to confirm task engagement. These analyses also demonstrated language-related activation in frontal and temporalparietal regions with left dominance in each TLE participant included in this study.

\section{Behavior PLS analyses}

Because our primary interest is in distributed patterns that correlated with percent retained, our measure of hippocampal integrity, we do not report in detail significant LVs that have a stable contribution from FSIQ only. We first report, in detail, PLS analyses that were performed across all patients regardless of side of seizure focus, and then briefly describe analyses performed on the left and right mTLE patients in separate groups.

\section{Mean fMRI signal in autobiographical memory and sentence completion}

Our first behavior PLS analysis focused on mean fMRI signal in the autobiographical memory and sentence completion tasks, and examined task-dependent correlations between our behavior 
measures (percent retained and FSIQ) and voxel signal mean throughout the brain. We identified one significant LV that showed regions in which increased activity supported good verbal memory performance (i.e., high percent retained scores), and had no relationship with general intelligence $(p<0.038$, explained covariance $=37 \%$; Fig. 1 ; Table 1 ). The correlation bar graph shows that there is no overlap in the $95 \%$ confidence intervals for percent retained scores and for FSIQ scores, indicating that the lack of contribution to this LV from FSIQ is stable (i.e., a stable zero contribution) (McIntosh and Lobaugh, 2004), and that the distributed pattern identified is specific to better verbal memory performance. Dominant positive saliences (indicating a positive correlation between increased brain activity during autobiographical memory and verbal memory performance) included left dorsomedial frontal gyrus (BA 10), right middle (BA 8 ), and superior frontal (BA 6) gyri, right middle temporal gyrus (BA 37), and right superior parietal lobule (BA 7). Dominant negative saliences (indicating a positive correlation between increased brain activity during sentence completion and verbal memory performance) included left lingual gyrus (BA 18) and right superior temporal gyrus (BA 42). Of note, medial temporal regions did not contribute to this pattern.

\section{SD of fMRI signal in autobiographical memory and sentence completion}

This analysis allowed us to identify task-dependent correlations between our behavior measures (percent retained and FSIQ) and voxel signal SD throughout the brain. We found one significant LV that showed regions in which increased variability during both tasks supported good verbal memory performance, and had no relationship with general intelligence $(p<0.001$, explained covariance $=68 \%$; Fig. 2; Table 2). The correlation bar graph shows that there is no overlap in the $95 \%$ confidence interval for percent retained scores and for FSIQ scores, suggesting that there is a stable zero contribution from FSIQ (McIntosh and Lobaugh, 2004). Here, dominant positive saliences (indicating a positive correlation between brain variability and memory performance) were located in medial temporal regions, including bilateral hippocampus and left parahippocampal gyrus (BA 28 and 30). Other temporal-lobe regions included left fusiform gyrus (BA 20), left inferior temporal gyrus (BA 37 ), right inferior temporal gyrus (BA 20 and 21), and right temporal pole (BA 38). Other regions in frontal cortex, cingulate, and subcortical structures are listed in Table 2. To further illustrate the relationship between brain signal variability and memory performance, we plotted the correlation between our most representative hippocampal voxel (i.e., the one with the highest bootstrap ratio) and percent retained (Fig. 3A,B). As illustrated, the r-squared for this voxel and percent retained in autobiographical memory is 0.38 , and in sentence completion is 0.26 .

\section{Mean $\mathrm{fMRI}$ signal in fixation, verb generation and category fluency}

We examined task-dependent correlations between our behavior measures (percent retained and FSIQ) and voxel signal mean throughout the brain, and identified distributed patterns of brain-behavior correlations. We identified two significant LVs. The first significant LV showed regions in which increased activity supported good verbal memory performance, and had no relationship with general intelligence ( $p=0.014$, explained covariance $=32 \%$; Fig. 4 ; Table 3 ). The dominant positive salience (indicating a positive correlation between increased brain activity during fixation and verbal memory performance) was located in left inferior temporal gyrus (BA 37). Dominant negative saliences

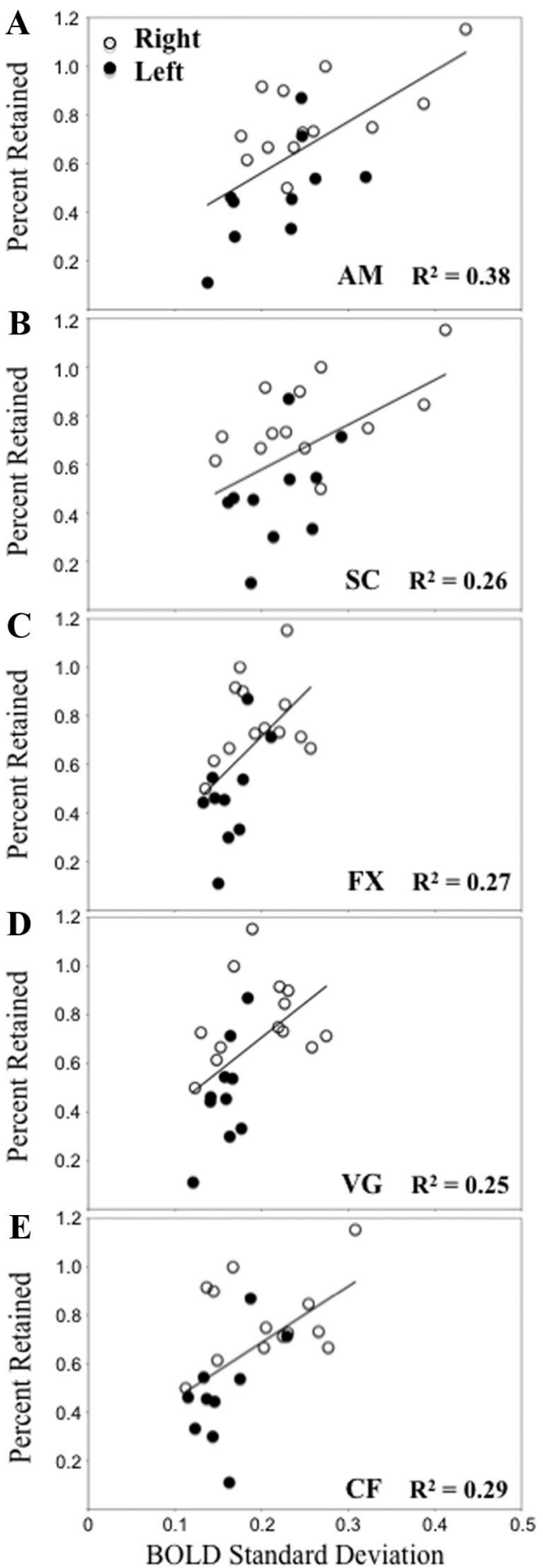

Figure 3. Correlation between BOLD SD in our most representative hippocampal voxel (i.e., the one with the highest bootstrap ratio) and percent retained from the autobiographical memory and sentence completion analysis $(\boldsymbol{A}, \boldsymbol{B} ; \mathrm{MNI}$ voxel coordinate $-18.0-33.0-6.0)$, and the fixation, verb generation and category fluency analysis $(\boldsymbol{C}-\boldsymbol{E}$; MNI voxel coordinate 24.0 $-33.0-6.0)$. FX, Fixation; $V G$, verb generation; $(F$, category fluency.

(indicating a positive correlation between increased brain activity during both word generation tasks and verbal memory performance) included left fusiform gyrus (BA 20), left posterior cingulate (BA 31), and left superior frontal gyrus (BA 8). As above, medial temporal regions did not contribute to this pattern.

The second significant LV showed regions in which increased activity supported high general intelligence, and had no relationship with verbal memory or with hippocampal activity $(p=0.01$, 
A

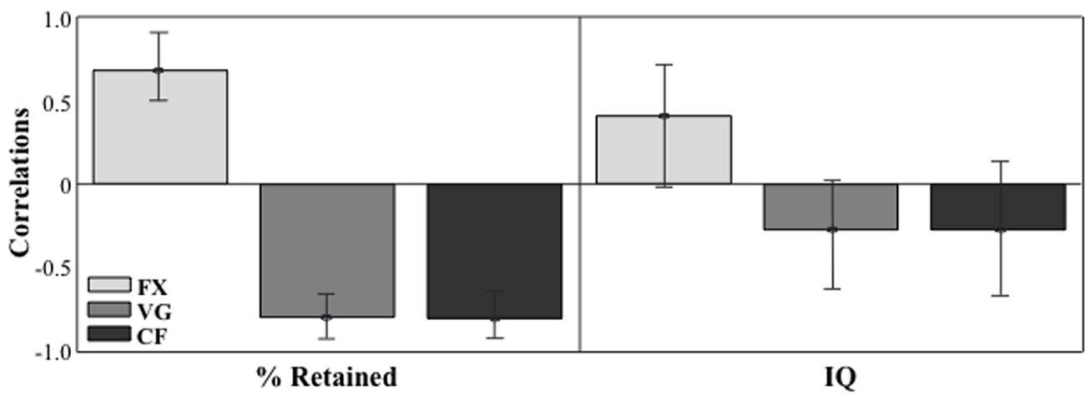

B

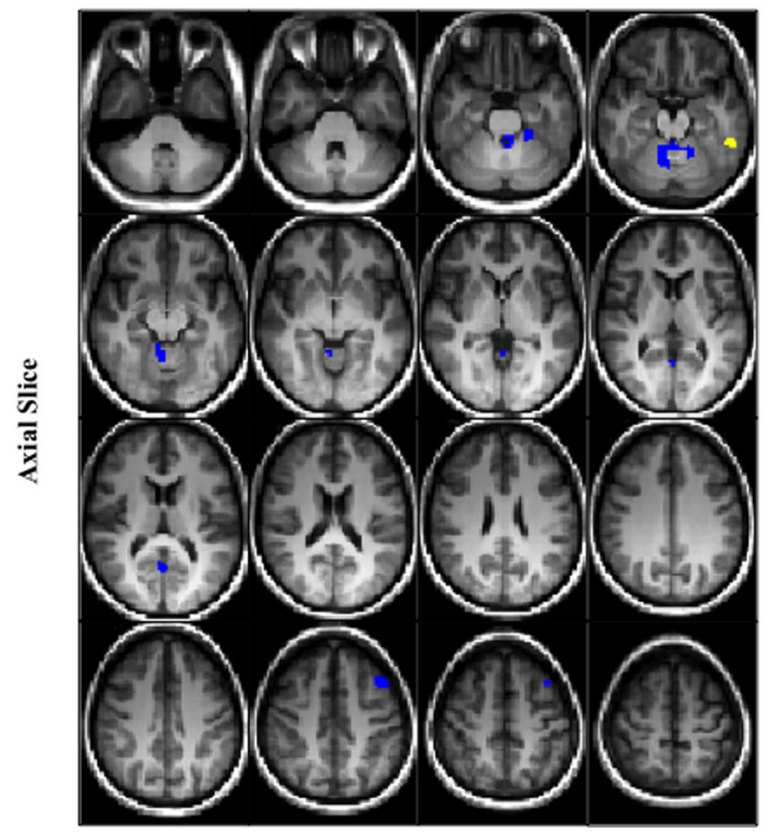

Figure 4. Correlation bar graph $(\boldsymbol{A})$ and singular image $(\boldsymbol{B})$ for LV1 from the mean fMRI signal in fixation, verb generation and category fluency behavior-PLS analysis. The correlation bar graph $(\boldsymbol{A})$ captures the task-dependent correlations between our behavior measures (percent retained and IQ) and the regions identified in the singular image. The error bars show the 95\% confidence interval derived from bootstrap estimation. The error bar crosses zero for IQ scores in all three tasks, indicating that there is no stable contribution from IQ to the pattern identified in the singular image. The singular image $(\boldsymbol{B})$ shows brain-behavior correlations for $\mathrm{FX}, \mathrm{VG}$, and $\mathrm{CF}$, displayed on axial slices in MNI atlas space. The brain is displayed according to radiological convention $(L=R)$. Regions highlighted in in yellow indicate a positive correlation between increased brain activity during $F X$ and better verbal memory performance. Regions highlighted in blue indicating a positive correlation between increased brain activity during VG and $\mathrm{SC}$ and better verbal memory performance.

Table 3. Local maxima for the mean fMRI signal in fixation, verb generation, and category fluency behavior PLS analysis

\begin{tabular}{llrrr}
\hline Region & $x$ & $y$ & \multicolumn{1}{l}{$z$} & \multicolumn{1}{c}{ BSR } \\
\hline Left inferior temporal gyrus (BA 37) & -57 & -39 & -15 & 4.79 \\
Left fusiform gyrus (BA 20) & -27 & -33 & -21 & -4.88 \\
Left superior frontal gyrus (BA 8) & -45 & 27 & 45 & -5.94 \\
Left posterior cingulate (BA 31) & -3 & -57 & 12 & -4.46 \\
\hline
\end{tabular}

Only voxels in supratentorial regions are reported. Regions indicate the gyral locations and $B A$ associated with the cluster peak.

$x, y$, and $z$ indicate voxel coordinates in MNI space. BSR represents each voxel's PLS parameter estimate divided by its SE.

explained covariance $=29 \%$ ). Because this LV does not inform our hypothesis, we will not discuss it further.

SD of fMRI signal in fixation, verb generation, and category fluency

This analysis identified one significant LV that showed regions in which increased variability supported good verbal memory per- formance, and had no relationship with general intelligence $(p<0.001$, explained covariance $=65 \%$; Fig. 5; Table 4). The correlation bar graph shows that there is no overlap in the $95 \%$ confidence interval for percent retained scores and for FSIQ scores, suggesting that there is a stable zero contribution from FSIQ (McIntosh and Lobaugh, 2004). As with the previous variability analysis, hippocampus and parahippocampal cortex were included among the dominant positive saliences that indicate a positive correlation between brain variability, here in all three task conditions, and memory performance. Several other regions in the temporal lobe were part of this distributed pattern, including left middle temporal gyrus (BA 37 and 39), bilateral temporal pole (BA 21 and 38), and left superior temporal gyrus (BA 21). Of interest, bilateral precuneus (BA 7), which is considered the "hub" of the default-mode network, was also part of this distributed pattern, as were several other cortical and subcortical regions listed in Table 4. To further illustrate the relationship between brain signal variability and memory performance, we plotted the correlation between the hippocampal voxel with the highest bootstrap ratio and percent retained (Fig. 3C-E). The r-squared for this voxel and percent retained in fixation is 0.27 , in verb generation is 0.25 , and in category fluency is 0.29 .

\section{Analyses performed with left and right mTLE patients in separate groups}

To ensure that our results do not change when mTLE patients are separated into two groups based on laterality of seizure onset, we replicated all above analyses in each patient group. The results were very similar to those described above, with the notable exception that in the variability analyses, dominant hippocampal saliences were no longer bilateral. The hippocampal voxels that were most reliably associated with percent retained were left lateralized for left mTLE patients, and right lateralized for right mTLE patients (Fig. 6).

\section{Discussion}

New computational and empirical research indicates that brain signal variability may be an important parameter for characterizing the functional integrity and processing capacity of neural systems (Nenadovic et al., 2008; McIntosh et al., 2008, 2010; Lippe et al., 2009; Deco et al., 2011; Garrett et al., 2011; Vakorin et al., 2011). Results of the current study with mTLE patients support this proposition. Across a variety of task conditions, we found that increased BOLD variability in a set of regions that included bilateral hippocampus was linked to better verbal memory retention measured outside the scanning session. This retention measure, derived from a standard neuropsychological test, is used clinically to determine the functional 
adequacy of the dominant MTL and predict the likelihood of postsurgical memory decline. Importantly, the increased variability was related specifically to a behavior that is strongly linked to functional integrity in the left hippocampus (Milner, 1971; Miller et al., 1993; Kilpatrick et al., 1997; Helmstaedter et al., 2009) and not to a measure of general intellectual functioning that reflects distributed as well as focal brain functioning. Furthermore, the link between signal in the hippocampus and individual differences in memory performance was only apparent for BOLD signal variability and was not observed when we assessed signal amplitude, even in conditions that have been shown to engage relevant medial temporal regions (i.e., autobiographical recall). Thus, differences in memory retention among patients were uniquely captured by intrinsic differences in brain signal variability in a distributed pattern of regions including bilateral MTL. The present finding expands on our previous intracranial EEG data, which demonstrated greater variability in the "healthy" versus epileptogenic hippocampus (Protzner et al., 2010), by now linking MTL variability to individual differences in a meaningful behavioral outcome.

In this study, we found a distributed set of brain regions that related mean signal specifically to our clinical measure of memory performance. However, these patterns did not include the hippocampus or other MTL structures (the critical area of interest for TLE), even for an autobiographical memory task known to rely on hippocampal integrity (Viskontas et al., 2000; St-Laurent et al., 2009; McAndrews, 2012) and to activate this region reliably in fMRI studies (Svoboda et al., 2006; Addis et al., 2007; McDermott et al., 2009). This finding is not in line with previous mTLE patient studies that have reported positive correlations between task-dependent regional activation in the hippocampus (or the MTL more broadly) and preoperative memory performance and/or postoperative memory decline (Rabin et al., 2004; Cheung et al., 2009; Bonelli et al., 2010; Guedj et al., 2011). However, there are several studies suggesting that straight-forward correlations between hippocampal activation and functional integrity of this region are not always the case. For example, Dickerson and Sperling (2008) have described increased MTL activity (relative to healthy controls) in individuals with very mild MCI and decreased activity in patients with more severe symptoms. In addition, we reported robust MTL activation in an individual during an episode of transient global amnesia during which memory formation and retrieval was dramatically impaired (Westmacott et al., 2008). Indeed, positive correlations between focal MTL activation and memory in patients with mTLE may not even be the modal pattern, although there

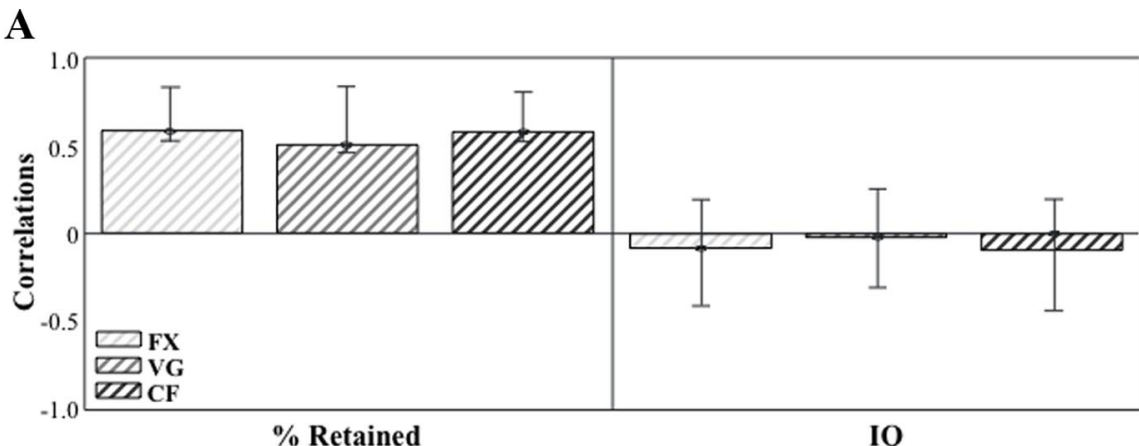

$\%$ Retained

IQ

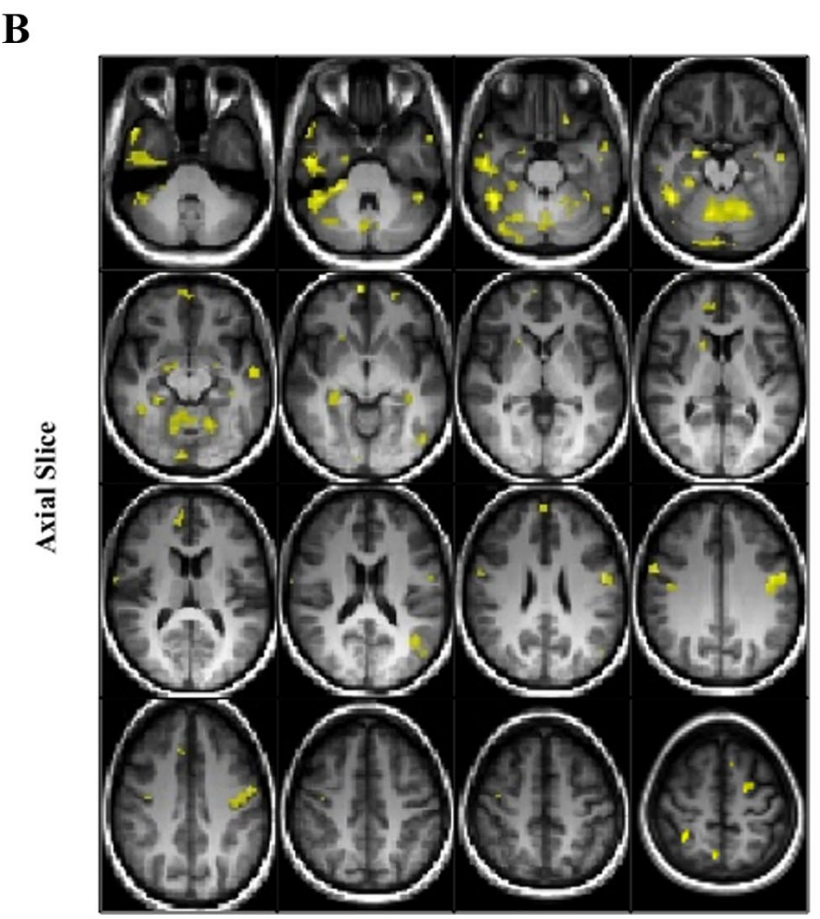

Figure 5. Correlation bar graph $(\boldsymbol{A})$ and singular image $(\boldsymbol{B})$ for LV1 from the SD of fMRI signal in fixation, verb generation and category fluency behavior-PLS analysis. The correlation bar graph $(\boldsymbol{A})$ captures the task-dependent correlations between ou radiological convention $(\mathrm{L}=\mathrm{R})$. Regions highlighted in yellow indicate a positive correlation between increased brain variability during all three tasks and better verbal memory performance.

are not yet enough relevant published data to derive firm conclusions.

In our view, there are many underspecified parameters in the relationship between task-dependent activation and processing integrity in mTLE patients. Among them are the nature and degree of structural damage, task engagement and performance, and duration of epilepsy. All of these may be crucial to predicting when imaging data are likely to reflect functional adequacy of the affected system versus compensation, or functional reserve of the rest of the brain (McAndrews and Cohn, 2012). Concerns like these have led to a strong interest in exploring the task-negative or resting state networks to characterize functional integrity. One feature that has shown promise in research on mild cognitive impairment and Alzheimer's Disease is the capacity to "toggle" between task-positive and task-negative networks. That is, the ability to "turn off" the default mode network (more precisely, the posterior cingulate cortex) when atten- 
Table 4. Local maxima for the standard deviation of fMRI signal in fixation, verb generation, and category fluency behavior PLS analysis

\begin{tabular}{lrrrr}
\hline Region & \multicolumn{1}{c}{ x } & \multicolumn{1}{c}{$z$} & BSR \\
\hline Right temporal pole (BA 21) & 45 & 21 & -30 & 7.33 \\
Right middle temporal gyrus (BA 20) & 48 & -12 & -24 & 6.84 \\
Right parahippocampal gyrus (BA 28) & 15 & -9 & -30 & 4.15 \\
Right hippocampus & 24 & -33 & -9 & 5.25 \\
Right amygdala & 18 & -3 & -15 & 6.96 \\
Right caudate nucleus & 12 & 18 & 9 & 4.67 \\
Right medial frontal gyrus (BA 8) & 6 & 33 & 39 & 4.61 \\
Right medial frontal gyrus (BA 9) & 36 & 51 & 12 & 4.79 \\
Right medial frontal gyrus (BA 10) & 3 & 66 & -3 & 6.30 \\
Right postcentral gyrus (BA 2) & 30 & -42 & 57 & 8.06 \\
Right precuneus (BA 7) & 3 & -60 & 57 & 7.02 \\
Left hippocampus & -42 & -27 & -9 & 4.13 \\
Left amygdala & -30 & -3 & -12 & 4.02 \\
Left middle temporal gyrus (BA 37) & -54 & -69 & 0 & 4.85 \\
Left middle temporal gyrus (BA 39) & -45 & -57 & 21 & 4.90 \\
Left superior temporal gyrus (BA 21) & -60 & -6 & -9 & 5.24 \\
Left inferior frontal gyrus (BA 47) & -21 & 27 & -21 & 5.35 \\
Left precentral gyrus (BA 6) & -48 & -3 & 33 & 5.81 \\
\hline
\end{tabular}

Only voxels in supratentorial regions are reported. Regions indicate the gyral locations and BA associated with the cluster peak.

$x, y$, and $z$ indicate voxel coordinates in MNI space. BSR represents each voxel's PLS parameter estimate divided by its $S E$
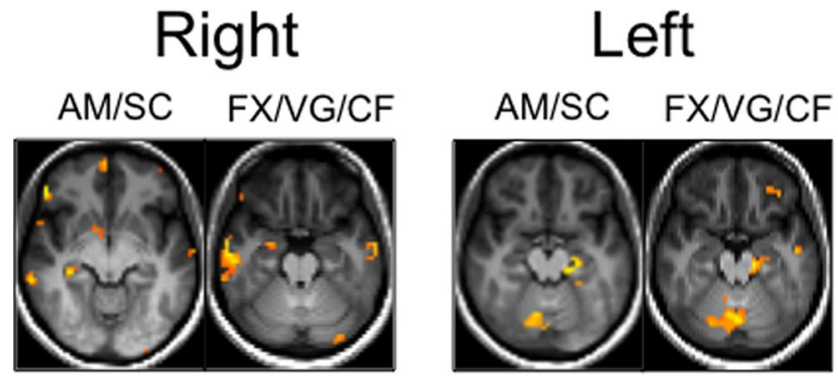

Figure 6. Axial slices, displayed according to radiological convention $(L=R)$, showing the hippocampal voxels that were most reliably associated with percent retained (i.e., the voxels with the highest bootstrap ratio) in analyses performed with left and right mTLE Patients in separate groups.

tion should be engaged to external stimuli during memory encoding has been shown to be related to clinical status in these patients (Petrella et al., 2007; Pihlajamäki and Sperling, 2009; Rombouts et al., 2009). In examining our own data, the task PLS for fixation and language tasks (data not shown) did not reveal many regions associated with the default mode network during fixation. Our behavioral PLS (Fig. 4) actually showed a modest positive relationship between inferior posterior cingulate activity during language tasks and percent retained. Although our current data do not speak to these activation or connectivity patterns involving task-negative networks, a recent study from our lab involving an overlapping cohort of TLE patients has shown connectivity between the more dorsal posterior cingulate (the hub of the default mode network) and hippocampus to account for a substantial proportion of variance in memory performance (McCormick et al., 2013). We contend that it will be an important challenge for future research to assess how well each of these metrics (task-related activation magnitude, downregulation of tasknegative regions of interest, measures of connectivity, and variability) fare in characterizing functional integrity of regions and networks.
One aspect of our results that deserves further commentary is our finding that variability in both left and right hippocampi were related to memory performance, despite the fact that verbal memory retention is considered to be strongly associated with left medial temporal dysfunction. Of interest, the correlations were strongest for distributed patterns involving the epileptogenic MTL in both groups, which suggests variability may be a sensitive indicator of MTL capacity to contribute to memory performance comparable to other metrics such as degree of atrophy. Clinical studies of memory outcomes in mTLE surgical patients emphasize that both functional adequacy of the ipsilesional MTL and functional reserve of the contralateral MTL and cortex may be equally important considerations in predicting surgical outcome for memory (Loring et al., 1992; Chelune, 1995). This finding would certainly comport with our variability findings when considering the entire cohort.

We found that the relationship of signal variability to memory performance was constant across a variety of activation paradigms, including fixation, word generation, and autobiographical memory. This is important for two reasons. First, it suggests that the variability measure can give the same answer across a range of cognitive challenges during scanning and thus it may be useful in many situations and patient populations. Second, it may be a more robust biomarker in that it appears to relate to functional integrity of clinically relevant brain structures in the individual, regardless of clinical condition/task engagement. Thus, although variability may scale with other independent variables, such as age (McIntosh et al., 2008,2010; Lippe et al., 2009; Garrett et al., 2010, 2011; Vakorin et al., 2011) and disease state (Protzner et al., 2010), it provides unique information regarding individual differences in functional integrity. This information, and the brain regions revealed as critical, is likely to vary with the particular biological context and system disorder. For example, if our main interest had been identifying regions associated with semantic knowledge in patients with semantic dementia, verbal IQ could be the criterion of interest and left frontal/temporal neocortex would be the neural substrate for interrogation. Extrapolating from computational modeling concepts, we might speculate that BOLD variability is a metric that reflects what the system (or regions of interest) is capable of doing whereas BOLD amplitude (or signal change in a specific contrast) reflects what the system is doing at any given moment of observation. That is, for clinical purposes, we would like to predict how the patient might be able to respond in a variety of cognitive challenges. Variability may capture this capacity to a greater extent than task-dependent activation during one circumscribed challenge. In this sense, variability may be similar to metrics derived from resting-state connectivity (McCormick et al., 2013). As it is indeed possible to derive all three measures from most fMRI studies with a sufficient number of observations, we would advocate systematic comparisons across patient populations to help us to further exploit the promise of BOLD as a useful and robust clinical biomarker.

\section{References}

Addis DR, Moscovitch M, McAndrews MP (2007) Consequences of hippocampal damage across the autobiographical memory network in left temporal lobe epilepsy. Brain 130:2327-2342. CrossRef Medline

Beckmann CF, Smith SM (2004) Probabilistic independent component analysis for functional magnetic resonance imaging. IEEE Trans Med Imaging 23:137-152. CrossRef Medline

Bonelli SB, Powell RH, Yogarajah M, Samson RS, Symms MR, Thompson PJ, Koepp MJ, Duncan JS (2010) Imaging memory in temporal lobe epilepsy: 
predicting the effects of temporal lobe resection. Brain 133:1186-1199. CrossRef Medline

Chelune GJ (1995) Hippocampal adequacy versus functional reserve: predicting memory functions following temporal lobectomy. Arch Clin Neuropsychol 10:413-432. CrossRef Medline

Chen XJ, Kovacevic N, Lobaugh NJ, Sled JG, Henkelman RM, Henderson JT (2006) Neuroanatomical differences between mouse strains as shown by high-resolution 3D MRI. Neuroimage 29:99-105. CrossRef Medline

Cheung MC, Chan AS, Lam JM, Chan YL (2009) Pre- and postoperative fMRI and clinical memory performance in temporal lobe epilepsy. J Neurol Neurosurg Psychiatry 80:1099-1106. CrossRef Medline

Cox RW (1996) AFNI: software for analysis and visualization of functional magnetic resonance neuroimages. Comput Biomed Res 29:162-173. CrossRef Medline

Deco G, Jirsa VK, McIntosh AR (2011) Emerging concepts for the dynamical organization of resting-state activity in the brain. Nat Rev Neurosci 12:43-56. CrossRef Medline

Dickerson BC, Sperling RA (2008) Functional abnormalities of the medial temporal lobe memory system in mild cognitive impairment and Alzheimer's disease: insights from functional MRI studies. Neuropsychologia 46:1624-1635. CrossRef Medline

Fox MD, Snyder AZ, Vincent JL, Corbetta M, Van Essen DC, Raichle ME (2005) The human brain is intrinsically organized into dynamic, anticorrelated functional networks. Proc Natl Acad Sci U S A 102:9673-9678. CrossRef Medline

Frank TD, Daffertshofer A, Beek PJ, Haken H (1999) Impacts of noise on a field theoretical model of the human brain. Physica D 127:233-249. CrossRef

Garrett DD, Kovacevic N, McIntosh AR, Grady CL (2010) Blood oxygen level-dependent signal variability is more than just noise. J Neurosci 30: 4914-4921. CrossRef Medline

Garrett DD, Kovacevic N, McIntosh AR, Grady CL (2011) The importance of being variable. J Neurosci 31:4496-4503. CrossRef Medline

Guedj E, Bettus G, Barbeau EJ, Liégeois-Chauvel C, Confort-Gouny S, Bartolomei F, Chauvel P, Cozzone PJ, Ranjeva JP, Guye M (2011) Hyperactivation of parahippocampal region and fusiform gyrus associated with successful encoding in medial temporal lobe epilepsy. Epilepsia 52:1100 1109. CrossRef Medline

Helmstaedter C, Wietzke J, Lutz MT (2009) Unique and shared validity of the "Wechsler logical memory test", the "California verbal learning test", and the "verbal learning and memory test" in patients with epilepsy. Epilepsy Res 87:203-212. CrossRef Medline

Hermann BP, Wyler AR, Somes G, Dohan FC Jr, Berry III AD, Clement L (1994) Declarative memory following anterior temporal lobectomy in humans. Behav Neurosci 108:3-10. CrossRef Medline

Kilpatrick C, Murrie V, Cook M, Andrewes D, Desmond P, Hopper J (1997) Degree of left hippocampal atrophy correlates with severity of neuropsychological deficits. Seizure 6:213-218. CrossRef Medline

Kovacević N, Henderson JT, Chan E, Lifshitz N, Bishop J, Evans AC, Henkelman RM, Chen XJ (2005) A three-dimensional MRI atlas of the mouse brain with estimates of the average and variability. Cereb Cortex 15:639645. CrossRef Medline

Lacadie CM, Fulbright RK, Rajeevan N, Constable RT, Papademetris X (2008) More accurate Talairach coordinates for neuroimaging using non-linear registration. Neuroimage 42:717-725. CrossRef Medline

Levine B, Kovacevic N, Nica EI, Cheung G, Gao F, Schwartz ML, Black SE (2008) The Toronto traumatic brain injury study: injury severity and quantified MRI. Neurology 70:771-778. CrossRef Medline

Lippe S, Kovacevic N, McIntosh AR (2009) Differential maturation of brain signal complexity in the human auditory and visual system. Front Hum Neurosci 3:48. CrossRef Medline

Loring DW, Meador KJ, Lee GP, King DW (1992) Amobarbital effects and lateralized brain function: the Wada test. New York: Springer.

McAndrews MP (2012) Remote memory and temporal lobe epilepsy. In A. Zeman, N. Kapur, and M. Jones-Gotman (Eds) Epilepsy and Memory, pp 227-243.Oxford: Oxford UP.

McAndrews MP and Cohn M (2012) Neuropsychology in temporal lobe epilepsy: influences from cognitive neuroscience and functional neuroimaging. Epilepsy Res Treat. 2012:925238. CrossRef Medline

McCormick C, Quraan M, Cohn M, Valiante TA, McAndrews MP (2013) Default mode network connectivity indicates episodic memory capacity in mesial temporal lobe epilepsy. Epilepsia 54:809-818. CrossRef Medline

McDermott KB, Szpunar KK, Christ SE (2009) Laboratory-based and autobiographical retrieval tasks differ substantially in their neural substrates. Neuropsychologia 47:2290-2298. CrossRef Medline

McIntosh AR, Gonzalez-Lima F (1991) Structural modeling of functional neural pathways mapped with 2-deoxyglucose: effects of acoustic startle habituation on the auditory system. Brain Res 547:295-302. CrossRef Medline

McIntosh AR, Lobaugh NJ (2004) Partial least-squares analysis of neuroimaging data: applications and advances. Neuroimage 23:S250-263. CrossRef Medline

McIntosh AR, Bookstein FL, Haxby JV, Grady CL (1996) Spatial pattern analysis of functional brain images using partial least-squares. Neuroimage 3:143-157. CrossRef Medline

McIntosh AR, Kovacevic N, Itier RJ (2008) Increased brain signal variability accompanies lower behavioral variability in development. PLoS Comput Biol 4:e1000106. CrossRef Medline

McIntosh AR, Kovacevic N, Lippe S, Garrett D, Grady C, Jirsa V (2010) The development of a noisy brain. Arch Ital Biol 148:323-337. Medline

Miller LA, Muñoz DG, Finmore M (1993) Hippocampal sclerosis and human memory. Arch Neurol 50:391-394. CrossRef Medline

Milner B (1971) Interhemispheric differences in the localization of psychological process in man. Brit Med Bull 27:272-277. Medline

Montez T, Poil SS, Jones BF, Manshanden I, Verbunt JP, van Dijk BW, Brussaard AB, van Ooyen A, Stam CJ, Scheltens P, Linkenkaer-Hansen K (2009) Altered temporal correlations in parietal alpha and prefrontal theta oscillations in early stage Alzheimer disease. Proc Natl Acad Sci U S A 106:1614-1619. CrossRef Medline

Monto S, Vanhatalo S, Holmes MD, Palva JM (2007) Epileptogenic neocortical networks are revealed by abnormal temporal dynamics in seizurefree subdural EEG. Cereb Cortex 17:1386-1393. CrossRef Medline

Nenadovic V, Hutchison JS, Dominguez LG, Otsubo H, Gray MP, Sharma R, Belkas J, Perez Velazquez JL (2008) Fluctuations in cortical synchronization in pediatric traumatic brain injury. J Neurotrauma 25:615-627. CrossRef Medline

O’Neil EB, Protzner AB, McCormick C, McLean DA, Poppenk J, Cate AD, Köhler S (2012) Distinct patterns of functional and effective connectivity between perirhinal cortex and other cortical regions in recognition memory and perceptual discrimination. Cereb Cortex 22:74-85. CrossRef Medline

Parish LM, Worrell GA, Cranstoun SD, Stead SM, Pennell P, Litt B (2004) Long-range temporal correlations in epileptogenic and nonepileptogenic human hippocampus. Neuroscience 125:1069-1076. CrossRef Medline

Pedhazur EJ (1982) Multiple regression in behavioral research: explanation and prediciton. Ed 2. New York: Holt, Reinhart, and Winston.

Petrella JR, Prince SE, Wang L, Hellegers C, Doraiswamy PM (2007) Prognostic value of posteromedial cortex deactivation in mild cognitive impairment. PLoS One 2:e1104. CrossRef Medline

Pihlajamäki M, Sperling RA (2009) Functional MRI assessment of taskinduced deactivation of the default mode network in Alzheimer's disease and at-risk older individuals. Behav Neurol 21:77-91. CrossRef Medline

Power JD, Barnes KA, Snyder AZ, Schlaggar BL, Petersen SE (2012) Spurious but systematic correlations in functional connectivity MRI networks arise from subject motion. Neuroimage 59:2142-2154. CrossRef Medline

Protzner AB, Valiante TA, Kovacevic N, McCormick C, McAndrews MP (2010) Hippocampal signal complexity in mesial temporal lobe epilepsy: a noisy brain is a healthy brain. Arch Ital Biol 148:289-297. Medline

Rabin ML, Narayan VM, Kimberg DY, Casasanto DJ, Glosser G, Tracy JI, French JA, Sperling MR, Detre JA (2004) Functional MRI predicts postsurgical memory following temporal lobectomy. Brain 127:2286-2298. CrossRef Medline

Rey A (1964) L'examen clinique en psychologie. Paris: Presses Univeritaires de France.

Rombouts SA, Damoiseaux JS, Goekoop R, Barkhof F, Scheltens P, Smith SM, Beckmann CF (2009) Model-free group analysis shows altered BOLD FMRI networks in dementia. Hum Brain Mapp 30:256-266. CrossRef Medline

Sampson PD, Streissguth AP, Barr HM, Bookstein FL (1989) Neurobehavioral effects of prenatal alcohol: part II. Partial least-squares analysis. Neurotoxicol Teratol 11:477-491. CrossRef Medline 
Sawrie SM, Martin RC, Gilliam F, Knowlton R, Faught E, Kuzniecky R (2001) Verbal retention lateralizes patients with unilateral temporal lobe epilepsy and bilateral hippocampal atrophy. Epilepsia 42:651-659. CrossRef Medline

Sherman EM, Wiebe S, Fay-McClymont TB, Tellez-Zenteno J, Metcalfe A, Hernandez-Ronquillo L, Hader WJ, Jetté N (2011) Neuropsychological outcomes after epilepsy surgery: systematic review and pooled estimates. Epilepsia 52:857-869. CrossRef Medline

St-Laurent M, Moscovitch M, Levine B, McAndrews MP (2009) Determinants of autobiographical memory in patients with unilateral temporal lobe epilepsy or excisions. Neuropsychologia 47:2211-2221. CrossRef Medline

Svoboda E, McKinnon MC, Levine B (2006) The functional neuroanatomy of autobiographical memory: a meta-analysis. Neuropsychologia 44: 2189-2208. CrossRef Medline

Talairach J, Tournoux P (1988) Co-planar stereotaxic atlas of the human brain. New York: Thieme Medical Publishers.

Tohka J, Foerde K, Aron AR, Tom SM, Toga AW, Poldrack RA (2008) Automatic independent component labeling for artifact removal in fMRI. Neuroimage 39:1227-1245. CrossRef Medline

Trenerry MR, Jack CR Jr, Ivnik RJ, Sharbrough FW, Cascino GD, Hirschorn
KA, Marsh WR, Kelly PJ, Meyer FB (1993) MRI hippocampal volumes and memory function before and after temporal lobectomy. Neurology 43:1800-1805. CrossRef Medline

Vakorin VA, Lipp é S, McIntosh AR (2011) Variability of brain signals processed locally transforms into higher connectivity with brain development. J Neurosci 31:6405-6413. CrossRef Medline

Viskontas IV, McAndrews MP, Moscovitch M (2000) Remote episodic memory deficits in patients with unilateral temporal lobe epilepsy and excisions. J Neurosci 20:5853-5857. Medline

Wechsler D (1999) Wechsler abbreviated scale of intelligence. New York: The Psychological Corporation.

Westmacott R, Silver FL, McAndrews MP (2008) Understanding medial temporal activation in memory tasks: evidence from fMRI of encoding and recognition in a case of transient global amnesia. Hippocampus 18 : 317-325. CrossRef Medline

Zhang D, Raichle ME (2010) Disease and the brain's dark energy. Nat Rev Neurol 6:15-28. CrossRef Medline

Zhang Z, Lu G, Zhong Y, Tan Q, Liao W, Wang Z, Li K, Chen H, Liu Y (2010) Altered spontaneous neuronal activity of the default-mode network in mesial temporal lobe epilepsy. Brain Res 1323:152-160. CrossRef Medline 